\title{
Longitudinal density monitor for the LHC
}

\author{
A. Jeff* \\ CERN, Geneva, Switzerland, and University of Liverpool, United Kingdom
}

\begin{abstract}
M. Andersen, A. Boccardi, S. Bozyigit, E. Bravin, T. Lefevre, A. Rabiller, and F. Roncarolo
CERN, Geneva, Switzerland

C. P. Welsch

Cockcroft Institute, Daresbury, United Kingdom
\end{abstract}

A. S. Fisher

SLAC, Menlo Park, California 94025, USA

(Received 6 February 2012; published 23 March 2012)

\begin{abstract}
The longitudinal density monitor (LDM) is primarily intended for the measurement of the particle population in nominally empty rf buckets. These so-called satellite or ghost bunches can cause problems for machine protection as well as influencing the luminosity calibration of the LHC. The high dynamic range of the system allows measurement of ghost bunches with as little as $0.01 \%$ of the main bunch population at the same time as characterization of the main bunches. The LDM is a single-photon counting system using visible synchrotron light. The photon detector is a silicon avalanche photodiode operated in Geiger mode, which allows the longitudinal distribution of the LHC beams to be measured with a resolution of 90 ps. Results from the LDM are presented, including a proposed method for constructing a 3-dimensional beam density map by scanning the LDM sensor in the transverse plane. In addition, we present a scheme to improve the sensitivity of the system by using an optical switching technique.
\end{abstract}

PACS numbers: 07.60.- j, 29.20.db, 29.27.Fh, 85.60.Gz

\section{INTRODUCTION}

The LHC is the world's largest particle accelerator and is designed to accelerate and collide protons or heavy ions [1]. So far protons have been accelerated up to $3.5 \mathrm{TeV}$ and lead ions up to $1.38 \mathrm{TeV}$ per nucleon.

The LHC rf cavities operate at $400 \mathrm{MHz}$ [2]. In the ultimate LHC filling pattern, 1 in 10 rf buckets will be filled with a bunch. Thus, the minimum bunch separation will be $25 \mathrm{~ns}$, with additional gaps left to account for the rise time of injection and extraction kicker magnets. This filling scheme is created in the LHC's injector complex which consists of a sequence of preaccelerators operating with different $\mathrm{rf}$ frequencies and filling schemes. Longitudinal matching of the beam throughout the complex requires sophisticated rf gymnastics in which longer bunches are split into a series of shorter bunches with some empty buckets between them [3]. Some particles can however enter these nominally empty buckets forming satellite bunches adjacent to the main bunches. In addition, off-momentum particles can escape from the rf buckets, especially during the modulation of the rf voltage for heavy

\footnotetext{
*adam.jeff@cern.ch
}

Published by the American Physical Society under the terms of the Creative Commons Attribution 3.0 License. Further distribution of this work must maintain attribution to the author(s) and the published article's title, journal citation, and DOI. ion injection. This debunched beam can later be recaptured to form ghost bunches, which spread throughout the LHC ring.

These satellite and ghost bunches can collide at the interaction points and create background noise for the experiments. In addition, they cause problems in the calibration of other instruments, principally for the measurement of absolute current and thus luminosity. Absolute current in the LHC is measured by the DC beam current transformers (DCCT). The fast beam current transformers (FBCT), which measure the bunch-by-bunch current, must be cross calibrated with the DCCT and in order to do this an allowance must be made for the fraction of the beam which is stored in the ghost bunches, to which the FBCT is blind [4]. In some cases, satellites can even cause machine protection problems, such as when satellites following a bunch train are kicked out by the injection of the next train. It is therefore very important to know the level and distribution of the ghost and satellite bunches.

Synchrotron radiation (SR) is an excellent tool for particle beam diagnostics as it is nondisruptive and carries information on both the transverse and longitudinal particle distribution. It is widely used in electron storage rings [5] where the SR intensities are very high. Since the SR power emitted is proportional to the fourth power of the relativistic $\gamma$, the intensity of SR from a proton beam is 13 orders of magnitude lower than an electron beam at the same energy and with the same radius of curvature. 


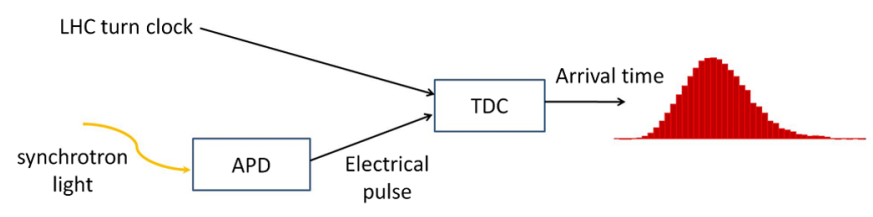

FIG. 1. Schematic of the longitudinal density monitor.

Nonetheless, synchrotron radiation has been used for diagnostics at the highest-energy proton accelerators using the edge effect [6-8].

A number of methods exist to convert synchrotron light into longitudinal profiles. As well as direct measurement with a photodiode and an oscilloscope, there are streak camera techniques [9], nonlinear mixing of the synchrotron light with light from a pulsed laser [10], or singlephoton counting (SPC) [11]. SPC is a relatively low-cost solution which is capable of measuring the full LHC ring with high dynamic range. SPC also compares favorably in this respect with electromagnetic measurements of the beam, such as wall current monitors or beam current transformers (BCT), where the dynamic range is usually limited due to noise and small mismatches in the impedance of the acquisition chain.

\section{PRINCIPLE OF THE DETECTOR}

Single-photon counting is used in order to achieve a high dynamic range. The system is illustrated schematically in Fig. 1. Each particle bunch passes the longitudinal density monitor (LDM) once every revolution (turn) and a photon could be detected from anywhere in the bunch, or not at all. In order to make a meaningful bunch profile, the data have to be collected over thousands of turns. The avalanche photodiode (APD) detects incoming photons and produces an electrical pulse. This is time stamped by a time to digital converter (TDC) and a histogram of arrival times is created. The longer the acquisition, the more counts are added to the histogram, and the higher the dynamic range of the measurement.

Synchrotron light is generated in a superconducting undulator and in a dipole magnet immediately downstream. The wavelength and intensity of synchrotron light are strongly dependent on the beam energy. Simulations using the SRW code [12] show that the dominant source of visible synchrotron light changes during acceleration from the undulator to the edge field of the dipole and then the main body of the dipole (see Table I). The undulator [13] was installed specifically for beam diagnostics and is necessary for measurements at injection since the dipole alone produces insufficient visible synchrotron light below $1.2 \mathrm{TeV}$ [14]. The undulator is always powered although it produces almost no visible light once the beam energy is greater than $1.5 \mathrm{TeV}$. Edge radiation from the entrance of the dipole is significant at intermediate energies from 1.2 to $2 \mathrm{TeV}$, while above $2 \mathrm{TeV}$ SR from the bend of the dipole is dominant.
TABLE I. Expected central frequency of SR emission at different beam energies. The spectrum of the dipole is very broad; since the undulator has only 2 periods its spectrum is also rather broad. Thus, even when both are centered in the UV there is still substantial visible light available.

\begin{tabular}{lcc}
\hline \hline & Dipole & Undulator \\
\hline $450 \mathrm{GeV}$ & $230 \mu \mathrm{m}$ & $610 \mathrm{~nm}$ \\
$3.5 \mathrm{TeV}$ & $485 \mathrm{~nm}$ & $10 \mathrm{~nm}$ \\
$7 \mathrm{TeV}$ & $60 \mathrm{~nm}$ & $2.5 \mathrm{~nm}$ \\
\hline \hline
\end{tabular}

An optical arrangement known as the beam synchrotron radiation telescope (BSRT) guides this light out of the beam pipe, focuses on the dominant source, and distributes it to the transverse profile cameras [15], to the abort gap monitor [16], and to the LDM, which receives $7 \%$ of the total light. The layout of the BSRT is shown in Fig. 2. The LDM line is equipped with two filter wheels allowing the attenuation to be controlled independently of the other BSRT instruments. The two filter wheels each have 6 positions and together they allow the attenuation to be varied between zero and optical density 7 in steps of not more than 0.3. The LDM detector is mounted on a remote controlled translation stage for alignment with $1.25 \mu \mathrm{m}$ resolution.

The detector used for the LDM is the photon detection module (PDM) from microphoton devices [17]. This is a silicon APD operated in the Geiger mode [18] in order to achieve sufficient sensitivity for single-photon counting. The detection efficiency is $\sim 35 \%$ averaged over the visible range, however the active area of the APD has a diameter of only $50 \mu \mathrm{m}$ and this leads to a substantial coupling loss. The time resolution is 50 ps FWHM. Like any Geigermode APD, the PDM has a dead time after each count, during which the detector is blind to further photons. The PDM uses an active quenching circuit with a dead time of $77 \mathrm{~ns}$. At the end of the dead time, the noise rate is increased due to afterpulsing. The integrated instrument response is illustrated in Fig. 3 and its effects on the signal are discussed in the following section. The PDM was chosen due to its excellent time resolution and ability to run at a high count rate. Unlike photomultiplier tubes (PMTs), APDs do not require a high voltage supply and are not damaged by strong illumination, giving them an advantage in terms of robustness and reliability. Nonetheless, the possibility of switching to the use of multichannel plate PMTs is under consideration due to their much larger active area.

The TDC used is an Acquiris TC890 (also called Agilent U1051A) [19]. The minimum bin width of $50 \mathrm{ps}$ is adequate considering the time resolution of the detector. A START pulse is provided at the revolution frequency by the LHC beam synchronous timing (BST) system. The BST optical signal is converted into an electrical pulse by a beam observation receiver (BOBR) module [20]. The output pulse of the BOBR has a jitter of approximately 


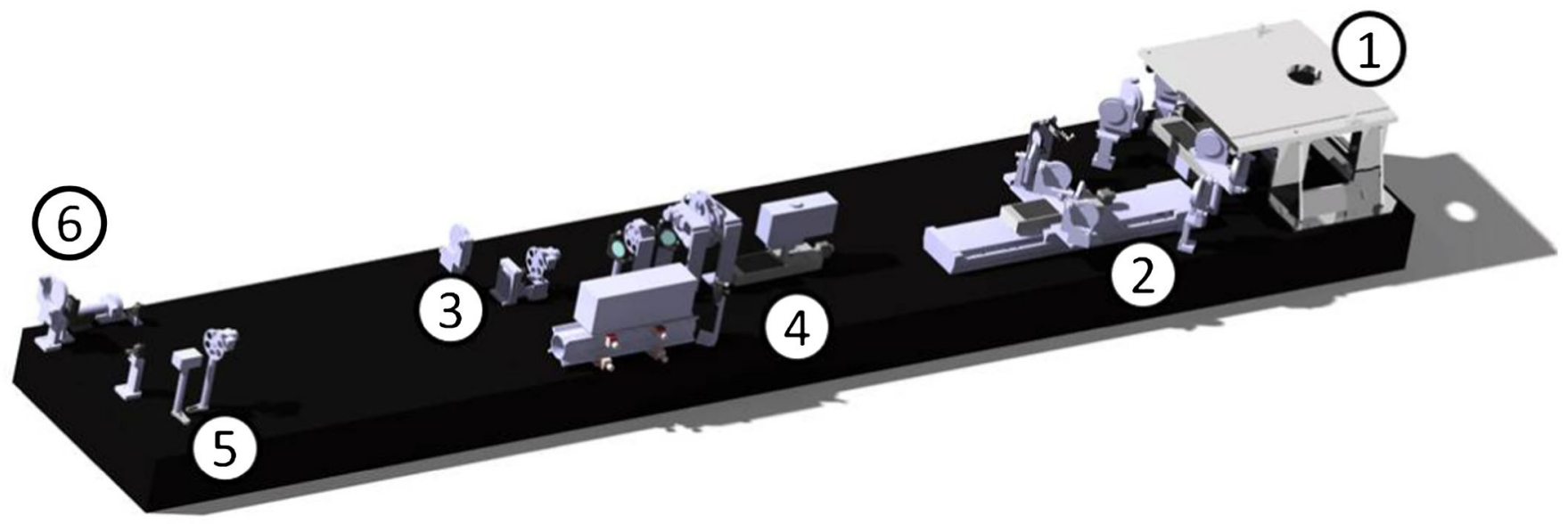

FIG. 2. Layout of the beam synchrotron radiation telescope (BSRT). The BSRT is located below the beampipe and the synchrotron light is directed down through the support (1) onto a motorized alignment mirror. It passes through a variable delay line or "trombone" (2) which is used to move the focus onto the dominant source of synchrotron light. The light is then split between the abort gap monitor (3), the transverse profile cameras (4), and the longitudinal density monitor (5). A calibration line (6) can be substituted for the synchrotron light.

$\sigma=65 \mathrm{ps}$. The STOP signals are the electrical pulses generated by the PDM. The TC890 is a multistop TDC so that the time stamps of many STOP pulses can be given relative to each START. The minimum separation of two STOPs is $15 \mathrm{~ns}$; since this is less than the dead time of the PDM it has no effect on the measurements.

\section{SIGNAL CORRECTION}

The dead time of the detector introduces a distortion to the measured pulse shape as well as providing an absolute upper limit to the count rate. The dead time is longer than the bunch length, so if a photon is detected from the first part of the pulse, the detector will be blind to any further photons. The measured pulse is thus skewed towards earlier time. In typical single-photon counting applications, the count rate is kept so low that there is a negligibly small

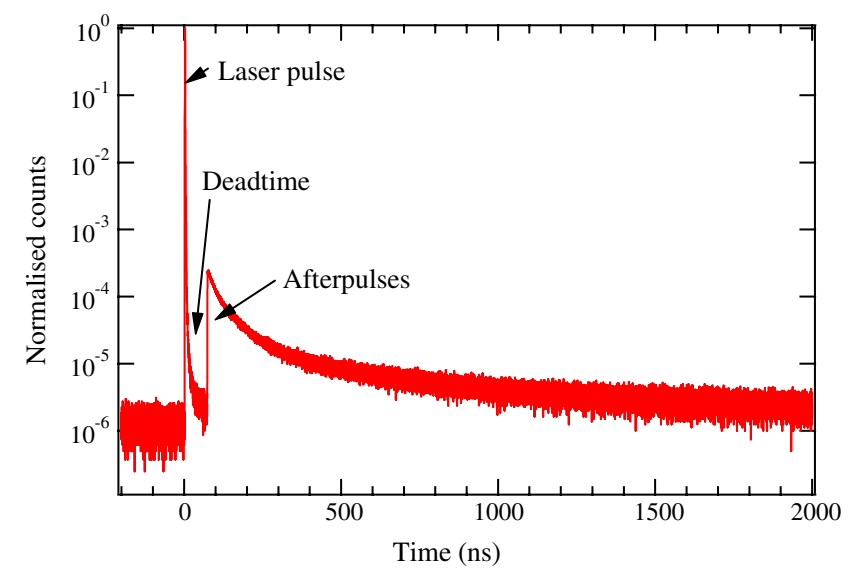

FIG. 3. Histogram of the instrument response function of the photon detection module (PDM). Measurement in the laboratory, integrated over $10^{8}$ cycles. probability of two photons arriving in the same pulse. However, in order to keep the integration time to a minimum it is desirable to have a higher count rate.

Consider the case of a small satellite bunch arriving after the main bunch, with a separation smaller than the dead time. The separation between main bunches is assumed to be longer than the dead time. It can be seen that if the light is too strongly attenuated, very few photons will arrive from the satellite bunch, while if the attenuation is too little the detector will almost always be blind to the satellite, having already seen a photon from the main bunch.

If the satellite bunch emits an average of $x$ photons per turn and the main bunch $n x$, then the probability of counting a photon from the satellite bunch is given by

$P($ photon from satellite $) \times P($ no photon from main bunch $)$

$$
=\left(1-e^{-x}\right) \times e^{-n x}
$$

since the emission of incoherent SR photons follows a Poissonian distribution. For large $n$ this can be shown to have a maximum at $n x=1$.

In order to optimize the sensitivity to satellite bunches, then, the attenuation should be set such that the average number of photons arriving per bunch is the reciprocal of the detection efficiency of the detector. A detector with no dead time would then count 1 photon per bunch on average. Since some photons arrive during dead time, however, the optimum raw count rate is 0.63 photons per bunch.

For the LHC running with $50 \mathrm{~ns}$ spacing, two bunches arrive within each dead time period. The optimum count rate per bunch is then half of the above.

In order to correct the number of counts in bin $i$, the total number of counts in the previous $77 \mathrm{~ns}$ of the histogram is found, and divided by the number of turns that were integrated over. This gives the probability that the APD 
was in dead time during bin $i$. A correction can then be calculated,

$$
C_{i}=\frac{x_{i}}{P(\text { ready })_{i}}=x_{i} \frac{N}{N-\sum_{j=i-d}^{i-1} x_{j}},
$$

where $x_{i}$ is the number of photons counted in bin $i$ over $N$ turns, $d$ is the dead time in bins, and $C_{i}$ is the number of photons which would be counted by a detector with no dead time [21].

This procedure was tested in the laboratory using a pulsed LED as the light source. The pulse shape was measured with a high arrival rate ( $\sim 2$ photons per pulse) and the correction applied. A neutral density filter with transmission of $0.01 \%$ was then placed in front of the detector and the pulse shape measured again. In this case the count rate was low enough to make the dead time effect negligible and no correction was applied. As shown in Fig. 4, the two measurements agree closely.

When the APD's operating voltage is restored it has an increased chance of producing a spontaneous avalanche caused by charge carriers trapped in the silicon. These correlated false counts or "afterpulses" are most likely to occur at the end of a dead time but can spread over several $\mu \mathrm{s}$ with probability decreasing further from the original count.

The afterpulses from subsequent avalanches are additive, that is, the probability of an afterpulse some time after two photons have been detected is the sum of the probabilities due to each photon individually (Fig. 5), so that the instrument response should be independent of the count rate. However, each avalanche causes a heating of the detector, and when operated at a high count rate this can exceed the capacity of the Peltier cooler and lead to a rise in temperature. The dark count and afterpulse probability

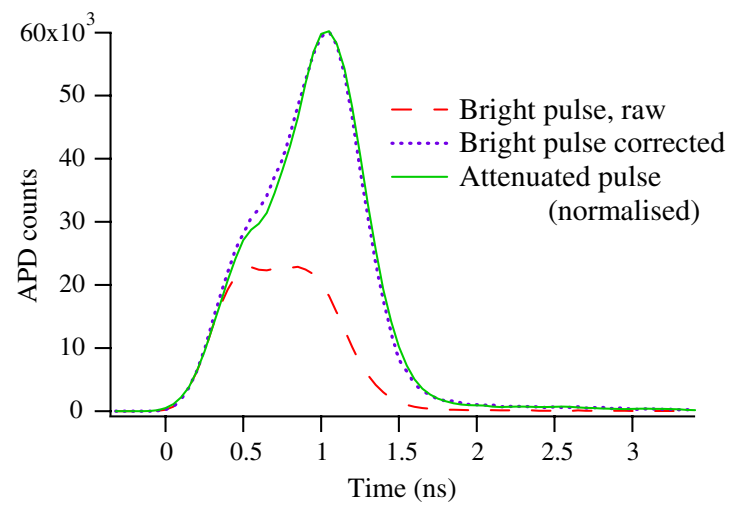

FIG. 4. Skew due to dead time can be compensated with an appropriate correction algorithm. The raw measurement is both reduced in magnitude and shifted towards the leading edge, due to the dead time effect. The correct pulse shape is restored after correction as can be seen by comparison with an attenuated pulse for which the dead time effect is negligible. The integral of the attenuated pulse has been normalized to that of the corrected bright pulse.

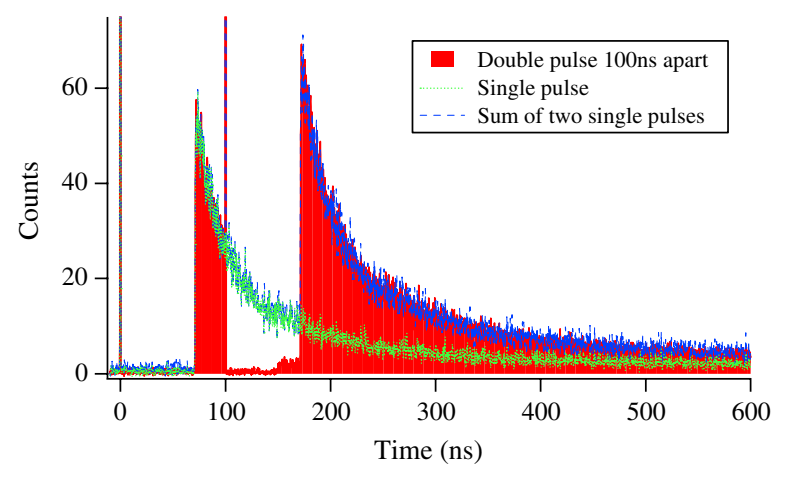

FIG. 5. The total probability of an afterpulse is the sum of the probabilities from each avalanche. Measurement in the lab using two laser pulses at time 0 and $100 \mathrm{~ns}$ (peaks at 400000 counts, not shown) compared to the sum of the counts from two normalized single pulses.

may depend on temperature [22] and this effect is under investigation.

The probability of an afterpulse occurring after any avalanche has been found experimentally to be approximately $3 \%$. The time distribution of the afterpulses is best fitted by multiple exponentials, with time constants ranging from $25 \mathrm{~ns}$ to $40 \mu \mathrm{s}$. The afterpulses can therefore be statistically eliminated by subtraction of a sequence of infinite impulse response (IIR) filters. This correction is applied to the LDM histogram and reduces the noise level by more than a factor of 10 . Because of uncertainty in determining the parameters of the IIRs, the afterpulses are not completely canceled, and some noise remains.

A third correction is applied to account for the possibility of two photons arriving during the same bin. The probability of this occurring is small but not negligible for the peak bins of each bunch. For an APD operated in Geiger mode, the arrival of multiple photons cannot be distinguished from a single photon, so the two photons produce only one count. The effect is to slightly flatten the peak. The arriving photons follow a Poissonian distribution, so their true arrival rate is

$$
P_{i}=-\ln \left(1-C_{i} / N\right)
$$

where $P_{i}$ is the number of photons emitted during bin $i$ on each turn, which is directly proportional to the particle density.

\section{RESULTS}

Measurements were taken with the LDM during both proton and lead ion runs. In the case of lead ions, the lower relativistic $\gamma$ means that the SR is almost entirely in the infrared at the injection energy of $177 \mathrm{GeV} / \mathrm{u}$, and measurements with the LDM were only possible above $350 \mathrm{GeV} / \mathrm{u}$. To illustrate the large dynamic range that can be achieved with correction, examples of typical profiles for both protons and heavy ions are shown in 
Fig. 6. Ghost bunches can be distinguished with a dynamic range close to $10^{5}$. A long integration time of several minutes is necessary in order to make a high dynamic range profile showing satellites, while profiles showing only main bunches can be made in approximately 10 seconds.

In the case of protons, almost all the satellites are spaced at $5 \mathrm{~ns}$ intervals, and so are thought to originate in the LHC injector chain where a lower rf frequency is used. In the case of heavy ions, small ghost bunches spaced at $2.5 \mathrm{~ns}$ are spread around the ring in addition to the larger satellites with $5 \mathrm{~ns}$ spacing found near to the main bunch. These ghost bunches are created in the LHC by the modulation of the rf voltage during injection to optimize capture for newly injected bunches, which led some particles from previously injected bunches to become debunched. These particles were subsequently recaptured once the rf voltage was again increased.

No other LHC instrument has the sensitivity to cross calibrate these satellite measurements. However, the satellite bunches generate collisions in the interaction points and this can be detected by the experiments. Satellite-main collisions, that is collisions caused by the crossing of a satellite from one beam with the main bunch of the other, occur off center in the detectors due to the different timing of the satellite bunches. The proportion of collisions at different locations within the detector can therefore be used to determine the relative satellite population. Satellite-satellite collisions occur both centered and off centered, but with negligible rate, since the probability of a collision is proportional to the product of the populations of the two colliding bunches.
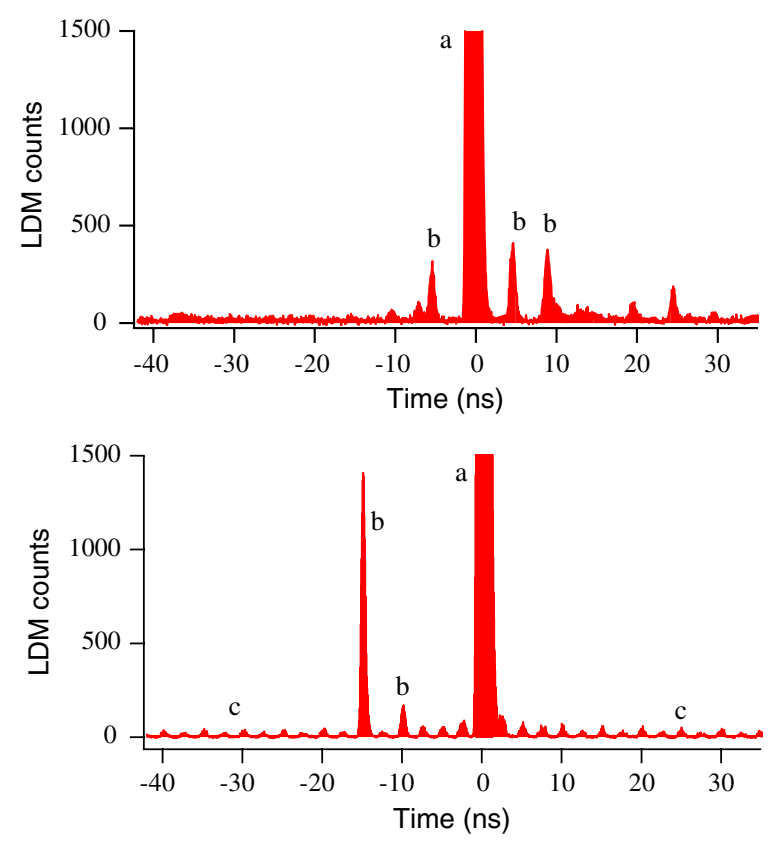

FIG. 6. Typical pattern of satellites in an LHC fill with protons (above) and with lead ions (below) showing (a) main bunch with peak at $1.3 \times 10^{5}$ counts, (b) satellites, and (c) ghost bunches.
The location of collisions within the experiment can be determined by direct imaging of the luminous region (vertex reconstruction) or by comparing the arrival time of collision products at detectors at each end of the experiment. In ATLAS the latter has been applied using the minimum bias trigger scintillators (MBTS) [23] and is shown in Fig. 7 for a lead ion fill in November 2010. This technique can only be applied to the heavy-ion run since the high pileup in the detectors during normal proton physics makes it impossible to determine the location of any one collision. For comparison with the LDM, the distribution of collisions within the ATLAS experiment is equivalent to the convolution of the longitudinal profiles of the two beams, averaged over all colliding bunches. At this time, the LDM was only operational on one beam, so the convolution of the LDM profile is performed with the same profile time reversed, that is assuming that the profile of both beams is the same.

Because of the dependence of the coupling efficiency on very precise steering of the light onto the LDM, it has been impossible to establish an absolute calibration factor. Since the main bunch currents are accurately measured by the BCTs, however, it is sufficient to measure the relative bunch to satellite populations. The LDM also measures the relative bunch-by-bunch populations and these can be compared to those measured by the fast BCTs in order to benchmark the LDM performance. They are found to agree to within $2 \%$. Similarly, the bunch lengths can be found by fitting a series of Gaussians to the LDM profile, and this is found to be in close agreement with the bunch length measured by the LHC wall current monitor [24], after subtracting in quadrature the LDM resolution of $90 \mathrm{ps}$.

The beam spot produced by the synchrotron light telescope is roughly Gaussian with a sigma between 100 and $500 \mu \mathrm{m}$ depending on the emittance of the beam. This

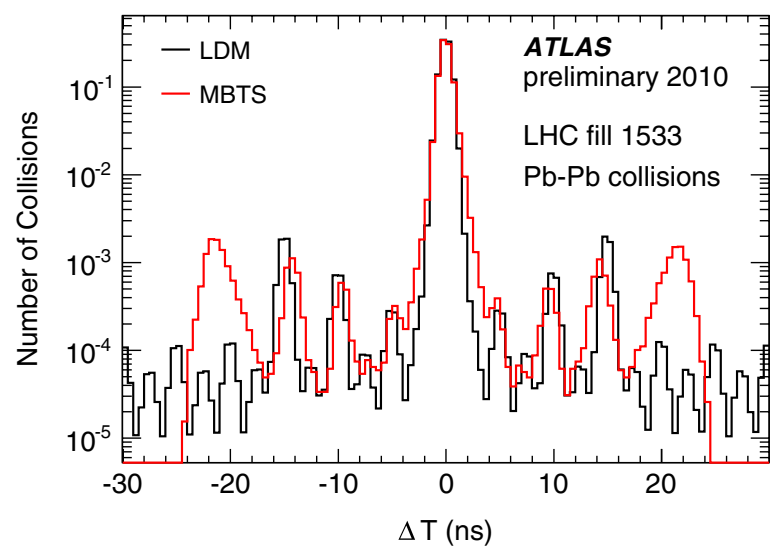

FIG. 7. Location of collisions in the ATLAS detector during the lead ion van der Meer scan in November 2010 (solid line), compared to the distribution derived from the LDM, assuming both beams had the same profile (dotted line). The peaks in the ATLAS data at $\pm 22 \mathrm{~ns}$ are due to beam losses outside the detector. 
reduces the coupling efficiency since only a fraction of the beam spot can be sampled by the $50 \mu \mathrm{m}$ active area of the detector. In addition, it creates a dependence of the coupling efficiency (and therefore the measured beam population) on the transverse size of the bunch, and therefore on the bunch emittance. If the detector is centered in the beam spot, then bunches with larger emittance will appear to have lower population. Conversely, if the detector position is away from the beam center, bunches with larger emittance will appear to have a larger population (Fig. 8). A minimum rate of change of coupling efficiency with emittance is found if the detector is placed 1 mean sigma away from the center.

This emittance dependency is clearly undesirable, especially since the emittance of the satellites is not well known. A change to the optical setup is proposed to eliminate the dependency. A small-angle diffuser is to be placed $0.5 \mathrm{~m}$ ahead of the detector, making a light spot in the detector plane whose size is largely independent of the beam size. A small focal length achromat lens is then used to condense the light spot and prevent too large a loss of coupling efficiency (Fig. 9).

However, the small active area of the detector with respect to the beam spot size also has a potential advantage. Since only a part of the transverse beam spot is sampled, a complete 3-dimensional map of the bunch population can be obtained by scanning the detector across the transverse plane. Around 1 hour is needed for a $10 \times 10$ point scan with adequate measurement of the main bunches, but not the satellites, and so the method is valid only on the assumption that the bunch shape is stable. Although the current typically decreases by a few per cent on this time scale, this can be normalized with reference to the BCTs.

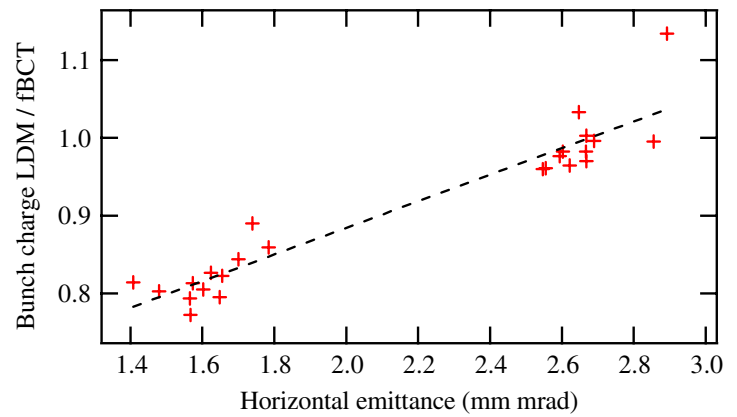

FIG. 8. Dependence of the measured bunch current on transverse bunch size for 24 bunches. The graph shows the ratio of the bunch charge measured by the LDM to the bunch charge measured by the fast beam current transformer (FBCT) against horizontal emittance, along with a linear fit. Measurement with beam during a dedicated run with 12 low-emittance and 12 highemittance bunches, with much larger variation in emittance than during normal operation. The LDM detector was off centered horizontally so that it received more light from bunches with larger horizontal beam size.

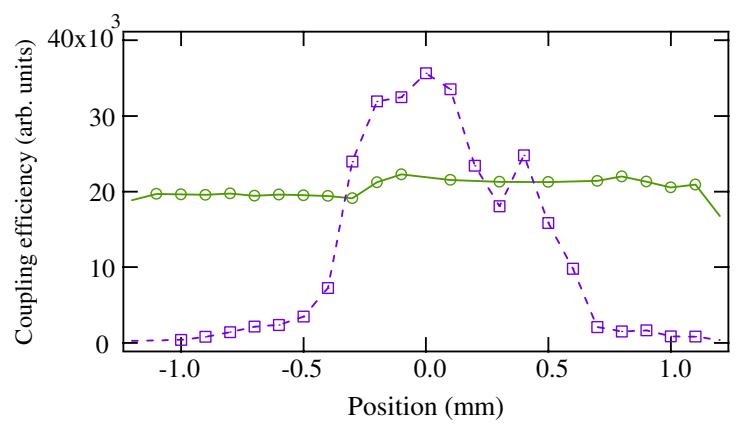

FIG. 9. Coupling efficiency vs position for the current optical setup (dashed line) and for the modified optical setup with diffuser and lens (solid line). Measurement performed in the lab using a pulsed laser as light source.

\section{VERY HIGH DYNAMIC RANGE SCHEME WITH OPTICAL GATING}

While the photon counting technique has been demonstrated to achieve a large dynamic range of $10^{5}$, this requires a long integration time of hundreds of seconds. The main limiting factor is the imperfect cancellation of afterpulsing. Since the integration time increases as the square of the dynamic range required, further dynamic range improvements by photon counting alone would need prohibitively long integration times. In addition, it would sometimes be desirable to make satellite and ghost bunch measurements on a much shorter time scale than is currently possible, for example, to validate the quality of a fill before proceeding to acceleration.

The limitation on dynamic range could be overcome by splitting the LDM into two systems, one with lower sensitivity which could make fast profiles of the main bunches, essentially the present system, and another which could make very sensitive measurements of the ghost and satellite bunches. This second system would use an optical gate to block out light from the main bunches. Much less attenuation would be necessary, so that more light would be received from the satellites, while the optical gate would prevent the detector being swamped by light from the main bunches. Results of a simulation into this technique are shown in Fig. 10.

An optical switching scheme is proposed using an electro-optic deflector [25]. The deflector consists of a crystal of nonlinear material which gives an angular deflection to the beam proportional to the electric field across the crystal. The deflector is driven with a $20 \mathrm{MHz}$ sine wave via a resonant circuit, producing a deflection of a few mrad. The detector is located a few meters away so that the deflection is translated into a transverse sweep of the beam spot. A lens is placed in front of the detector so that the light is directed onto the detector regardless of the deflection angle. There is now no modulation of the signal but the temporal profile of the beam has been mapped on the 


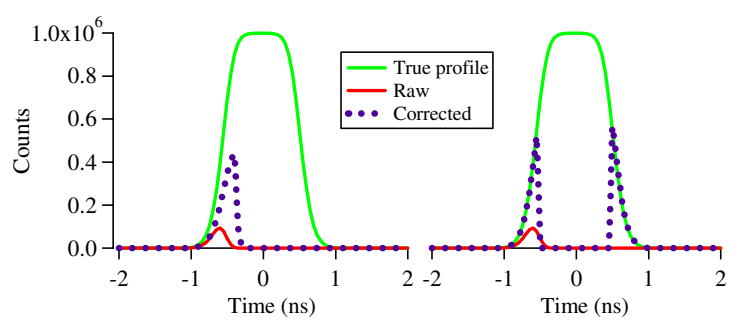

FIG. 10. Results from a simulation into an optically gated longitudinal density monitor. The attenuation is reduced so that on average 100 photons arrive from each bunch. Without gating, the probability of reaching the middle of the bunch without having detected a photon is negligible, and the correction algorithm cannot reconstruct the true bunch shape (left). By gating the main part of the bunch, the count rate is reduced to an acceptable level without requiring attenuation, and the shape of the bunch tails, and any following satellites, can be accurately reconstructed (right).

transverse plane of the lens. A mask placed in front of the lens can be used to block out part of the profile (Fig. 11). In this case, the central line would be blocked, since the greatest transverse speed of the beam spot, and therefore the fastest switching speed, is achieved in the center of the deflection. The deflecting voltage must be carefully synchronized so that the light from the bunch arrives when there is no deflection. The frequency of the deflecting sine wave is $20 \mathrm{MHz}$, half the bunch frequency, since the beam spot is swept over the central line twice in each period.

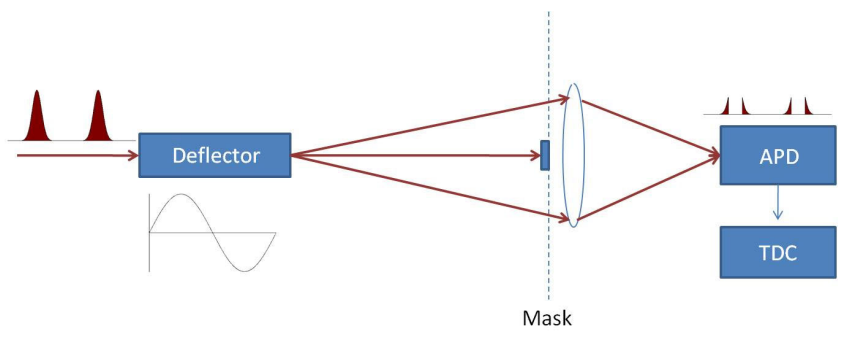

FIG. 11. Schematic of the optical switching scheme.

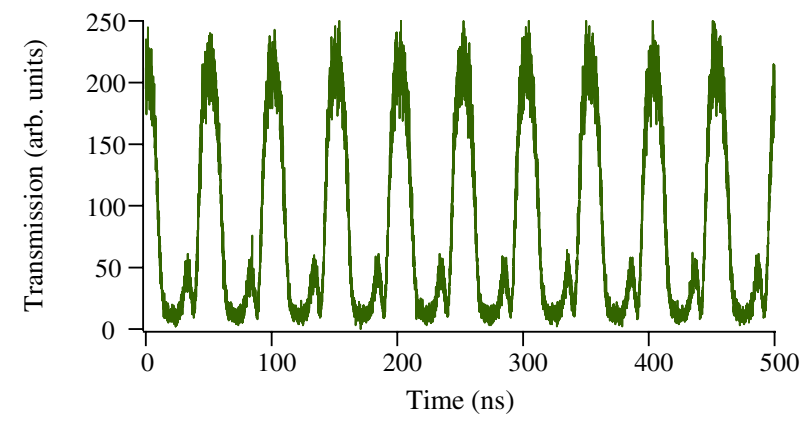

FIG. 12. Optical gating efficiency for preliminary test of the deflector scheme.
Preliminary testing of the switching scheme has been carried out in the lab. However, a suitable driver for the deflector was not available, and less than half the required deflection was realized. A simplified setup was used, with the modulation then at $20 \mathrm{MHz}$. The maximum extinction ratio was only 1:20 and was limited primarily by the small deflection angle and by diffraction of the beam spot due to the deflector aperture. Nonetheless, the results illustrate the principle and are shown in Fig. 12.

A simpler system using a fast Pockels cell with crossed polarizers has been demonstrated elsewhere [26] but has a limited repetition rate, which would counter the benefit in integration time achieved by blocking the main bunch.

\section{CONCLUSION}

It is shown that a photon-counting method can produce longitudinal profiles of the LHC beams with high dynamic range and good time resolution. Bunch-by-bunch measurement of all bunches in the machine is made simultaneously. Silicon APDs operated in Geiger mode are used for photon detection. The dynamic range of the system is largely limited by the response of these APDs, in particular by the dead time and by afterpulsing. Correction of the signal for these effects substantially increases the dynamic range. A further improvement in dynamic range could be achieved if an optical gating could be applied to the signal, and a scheme is proposed to implement this using electrooptic deflection.

The small active area of the APDs causes difficulties with coupling stability and emittance dependence, but also opens the possibility of 3-dimensional bunch shape measurements by scanning the detector over the transverse plane.

The longitudinal density monitor has been proven against established instruments such as the beam current transformers and wall current monitors. The population of satellites close to the colliding bunches has also been confirmed by data from the experiments.

The LDM is now widely used in LHC operation and plays an important part in the van der Meer scan procedure which is used for absolute luminosity calibration by the LHC experiments. The LDM is the primary tool for the quantification of ghost and satellite bunches, which can be measured with a dynamic range of $10^{5}$.

\section{ACKNOWLEDGMENTS}

Thanks to Beate Heinemann of ATLAS and to others from the LHC experiments for their satellite analysis. A. Jeff is funded by Ditanet, a Marie Curie Action of the E.U., Contract No. PITN-GA-2008-215080. A. S. Fisher's work was partly supported by the U.S. DOE, through the U.S. LHC Accelerator Research Program (LARP) and Contract No. DE-AC02-76SF00515 (SLAC). 
[1] O. Bruning, P. Collier, P. Lebrun, S. Myers, R. Ostojic, J. Poole, and P. Proudlock, LHC Design Report (CERN), 2004.

[2] D. Boussard and T. Linnecar, LHC Project Report No. 316, 1999.

[3] M. Benedikt, R. Cappi, M. Chanel, R. Garoby, M. Giovannozzi, S. Hancock, M. Martini, E. Métral, G. Metral, K. Schindl, and J.-L. Vallet, in Proceedings of HEACC, Tsukuba, 2001, Vol. 11, DOI 10.1063/ 1.2401426.

[4] J. Gras, D. Belohrad, M. Ludwig, P. Odier, and C. Barschel, in Proceedings of IPAC, San Sebastian, 2011, pp. 1395-1397 [http://lss.fnal.gov/archive/2006/pub/ fermilab-pub-06-091-ad.pdf].

[5] Shiro Takano, in Proceedings of the IPAC'10 Conference, Kyoto, Japan (ICR, Kyoto, 2010), pp. 2392-2396.

[6] G. Kube, G. Priebe, C. Wiebers, and K. Wittenburg, in Proceedings of the 12th Beam Instrumentation Workshop, Batavia, Illinois, 2006 (AIP, Melville, New York, 2006), pp. 374-383.

[7] R. M. Thurman-Keup, in Proceedings of the 12th Beam Instrumentation Workshop, Batavia, Illinois, 2006 (Ref. [6]), pp. 364-373.

[8] R. Bossart, J. Bosser, L. Burnod, E. d'Amico, G. Ferioli, J. Mann, and F. Meot, Nucl. Instrum. Methods 184, 349 (1981).

[9] C.P. Welsch, H.H. Braun, E. Bravin, R. Corsini, S. Dobert, T. Lefevre, F. Tecker, P. Urschutz, B. Buonomo, O. Coiro, A. Ghigo, and B. Preger, JINST 1, P09002 (2006).

[10] J.-F. Beche, J. Byrd, S. De Santis, P. Denes, M. Placidi, W. Turner, and M. Zolotorev, in Proceedings of BIW, Knoxville, 2004, pp. 112-119, DOI 10.1063/1.1831137.

[11] W. Becker, Advanced Time-correlated Single Photon Counting Techniques (Springer, Berlin Heidelberg, 2005).
[12] O. Chubar and P. Elleaume, in Proceedings of the 6th European Particle Accelerator Conference, Stockholm, 1998 (IOP, London, 1998), pp. 1177-1179.

[13] R. Maccaferri, M. Facchini, R. Jung, D. Tommasini, and W. Venturini Delsolaro, in Proceedings of the 9th European Particle Accelerator Conference, Lucerne, 2004 (EPS-AG, Lucerne, 2004), pp. 1630-1632.

[14] A. Jeff, S. Bart Pedersen, A Boccardi, E. Bravin, A. S. Fisher, A. Guerrero, T. Lefevre, and A. Rabiller, in Proceedings of SPIE, Brussels, 2010, pp. 77261J1-10, DOI 10.1117/12.853848.

[15] T. Lefevre, E. Bravin, G. Burtin, A. Fisher, A. Guerrero, A. Jeff, A. Rabiller, and F. Roncarolo, in Proceedings of the IPAC'10 Conference, Kyoto, Japan (Ref. [5]), pp. 1104-1106.

[16] T. Lefevre, S. Bart Pedersen, A. Boccardi, E. Bravin, A. Jeff, A. Goldblatt, F. Roncarolo, and A.S. Fisher, in Proceedings of the IPAC'10 Conference, Kyoto, Japan (Ref. [5]), pp. 2863-2865.

[17] http://www.microphotondevices.com.

[18] A. D. Renker, Nucl. Instrum. Methods 567, 48 (2006).

[19] http://www.agilent.com.

[20] J.-J. Savioz, CERN Report No. LHC-BOBR-ES-0001, 2003.

[21] E. Bravin, CERN Report No. AB-2006-017 BI, 2006.

[22] D. Renker and E. Lorenz, JINST 4, P04004 (2009).

[23] T. Martin, JINST 5, C12051 (2010).

[24] A. Jeff, A. Boccardi, E. Bravin, A. S. Fisher, T. Lefevre, A. Rabiller, F. Roncarolo, and C. P. Welsch, Nucl. Instrum. Methods Phys. Res., Sect. A 659, 549 (2011).

[25] Jun Kang, Wei Zhang, Hui Wei, Shaohe Chen, and Jianqiang Zhu, Chin. Optic. Lett. 4, 3 (2006) [http:// www.opticsinfobase.org/col/abstract.cfm?id=88557].

[26] Kazuhiro Tamura, Spring-8 annual report (1998), pp. 146-147. 